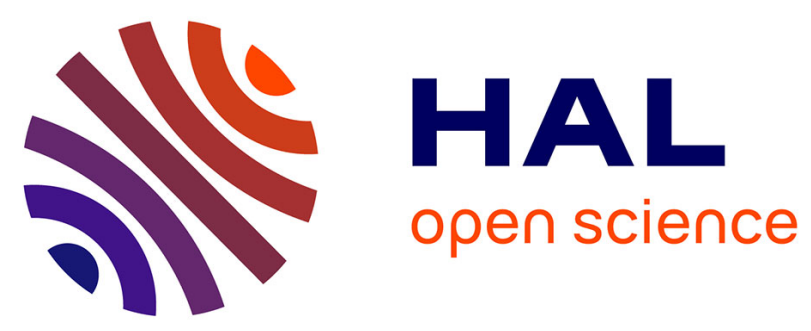

\title{
The impact of inertial effects on solute dispersion in a channel with periodically varying aperture
} Jeremy Bouquain, Yves Méheust, Diogo Bolster, Philippe Davy

\section{To cite this version:}

Jeremy Bouquain, Yves Méheust, Diogo Bolster, Philippe Davy. The impact of inertial effects on solute dispersion in a channel with periodically varying aperture. Physics of Fluids, 2012, 28 (4), pp.083602. 10.1063/1.4747458 . insu-00745643

\section{HAL Id: insu-00745643 \\ https://hal-insu.archives-ouvertes.fr/insu-00745643}

Submitted on 6 Nov 2012

HAL is a multi-disciplinary open access archive for the deposit and dissemination of scientific research documents, whether they are published or not. The documents may come from teaching and research institutions in France or abroad, or from public or private research centers.
L'archive ouverte pluridisciplinaire HAL, est destinée au dépôt et à la diffusion de documents scientifiques de niveau recherche, publiés ou non, émanant des établissements d'enseignement et de recherche français ou étrangers, des laboratoires publics ou privés. 


\title{
The impact of inertial effects on solute dispersion in a channel with periodically varying aperture
}

\author{
J. Bouquain, ${ }^{1, a)}$ Y. Méheust, ${ }^{1, b)}$ D. Bolster, ${ }^{2}$ and P. Davy ${ }^{1}$ \\ ${ }^{1}$ Geosciences Rennes (UMR CNRS 6118), Université Rennes 1, Campus de Beaulieu, \\ 35042 Rennes Cedex, France \\ ${ }^{2}$ Department of Civil Engineering and Geological Sciences, University of Notre Dame, \\ Notre Dame, Indiana 46556 USA
}

(Received 9 May 2012; accepted 26 July 2012; published online 30 August 2012)

\begin{abstract}
We investigate solute transport in channels with a periodically varying aperture, when the flow is still laminar but sufficiently fast for inertial effects to be nonnegligible. The flow field is computed for a two-dimensional setup using a finite element analysis, while transport is modeled using a random walk particle tracking method. Recirculation zones are observed when the aspect ratio of the unit cell and the relative aperture fluctuations are sufficiently large; under non-Stokes flow conditions, the flow in non-reversible, which is clearly noticeable by the horizontal asymmetry in the recirculation zones. After characterizing the size and position of the recirculation zones as a function of the geometry and Reynolds number, we investigate the corresponding behavior of the longitudinal effective diffusion coefficient. We characterize its dependence on the molecular diffusion coefficient $D_{\mathrm{m}}$, the Péclet number, the Reynolds number, and the geometry. The proposed relation is a generalization of the well-known Taylor-Aris relationship relating the longitudinal dispersion coefficient to $D_{\mathrm{m}}$ and the Péclet number for a channel of constant aperture at sufficiently low Reynolds number. Inertial effects impact the exponent of the Péclet number in this relationship; the exponent is controlled by the relative amplitude of aperture fluctuations. For the range of parameters investigated, the measured dispersion coefficient always exceeds that corresponding to the parallel plate geometry under Stokes conditions; in other words, boundary fluctuations always result in increased dispersion. The transient approach to the asymptotic regime is also studied and characterized quantitatively. We show that the measured characteristic time to attain asymptotic conditions is controlled by two competing effects: (i) the trapping of particles in the near-immobile zone and, (ii) the enhanced mixing in the central zone where most of the flow takes place (mainstream), due to its thinning. (C) 2012 American Institute of Physics. [http://dx.doi.org/10.1063/1.4747458]
\end{abstract}

\section{INTRODUCTION}

Ever since Taylor's seminal work ${ }^{1}$ where he demonstrated that the transport of a solute in an axisymmetrical shear flow was effectively reduced to a one-dimensional dispersion process by using a longitudinal effective dispersion coefficient, the concept of effective dispersion has proven enormously useful and popular across a wide range of fields and applications. This includes, but is not limited to micro fluidic systems, ${ }^{2,3}$ nutrient transport in bloodflow, ${ }^{4,5}$ single and multiphase transport in porous media, ${ }^{6-12}$ and transport in groundwater systems. ${ }^{13-16}$

The basic idea behind Taylor dispersion is simple. At "asymptotic" times, which are times when the solute has sampled by diffusion the full variability of the flow velocities, gradients of solute in the direction transverse to the flow direction can be considered negligible. At these times spreading of the

\footnotetext{
a) jeremy.bouquain@univ-rennes1.fr.

b) yves.meheust@univ-rennes1.fr.
} 
plume occurs only in the longitudinal direction and can be described by a one-dimensional advectiondiffusion model. The corresponding diffusive term features an effective dispersion coefficient that contains the longitudinal molecular diffusion but results mostly from the interaction between the transverse heterogeneity of the velocity field and the molecular diffusion in the transverse direction. The results of the earliest works, which addressed the axisymmetrical (cylindrical tube) geometry, ${ }^{1,17}$ were later generalized to other geometries including the parallel plate, ${ }^{18,19}$ and the principles hold for more complex systems. At times earlier than these "asymptotic" times, the behavior is more complicated as the rate of spreading of the plume and mixing are not the same. ${ }^{14,20} \mathrm{~A}$ variety of works exist studying these pre-asymptotic times for the cylindrical- or parallel plate-configurations, ${ }^{21-23}$ and also when density-driven coupling of flow and transport is present. ${ }^{24}$ However, all these prior studies address advecting flows with no significant inertial effects (Stokes flow).

In many applications of practical interest, the relevant channels do not have constant aperture. However, using slightly more complicated approaches the notion of Taylor dispersion can still readily be applied using what has been coined generalized Taylor dispersion theory, which is based on the method of local moments. ${ }^{6}$ Using such approaches, many authors have shown that deviation from parallel smooth boundaries can significantly alter behavior, ${ }^{25}$ leading to relative increases, ${ }^{26-29}$ or even decreases ${ }^{7,30,31}$ in the effective dispersion.

In most studies for flow through porous media it is reasonable to assume small Reynolds numbers $R e \leq \mathrm{o}(1)$. Thus it is common practice to assume that flow is governed by the Stokes equations where inertial effects are neglected. While this is very often a reasonable assumption, ${ }^{32}$ a variety of practical situations exist where the Reynolds number can become of order unity and larger, so that inertial effects are no longer negligible. Practical examples include flow through fractures with large aperture $^{33-36}$ and flows where the viscosity can be small such as carbon sequestration where the viscosity of supercritical $\mathrm{CO}_{2}$ can be one or two orders of magnitude smaller than that of water. ${ }^{37}$

Increased inertial effects play an interesting role on the structure of the flow. ${ }^{38,39}$ In particular. they lead to the presence of recirculation zones. ${ }^{33}$ Such recirculation zones can actually also occur in Stokes flow (see examples in Refs. 7, 16,40, and 41 as well as Ref. 42 for an account of why it occurs), but under increased inertial effects they develop under much weaker geometrical constraints and also exhibit less symmetry than in Stokes flows. These recirculation zones represent low velocity regions that can have a significant impact on effective solute transport and, in particular, on the asymptotic dispersion, both for reactive ${ }^{32}$ and inert solute transport, ${ }^{7}$ depending on typical mass transfer time scales. ${ }^{43-45}$

In this work, considering an idealized pore geometry, we focus on flow regimes at Reynolds numbers larger than 1, where inertial effects become significant. However, we do not consider situations where the Reynolds number becomes sufficiently large for the flow to become turbulent. We consider the evolution in size of the recirculation zones with increasing Reynolds number and investigate what effect this has on the pre-asymptotic transport and ultimately on asymptotic longitudinal dispersion. We compute the two-dimensional flow field using a finite-element model and the solute transport based on a random walk method. We first characterize the flow and transport from a phenomenological point of view and then examine quantitatively how the time derivative of the second centered moment of the solute concentration field evolves in time, in particular, how its asymptotic value scales with the Reynolds and Péclet numbers, and how that scaling depends on the geometry.

The paper is organized as follows: we describe the geometry, the mathematical basis and the numerical implementation of our simulations in Sec. II; the results are presented in Sec. III, and discussed in Sec. IV.

\section{METHODS}

\section{A. Geometry definition}

We define a two-dimensional geometry with a sinusoidal wall boundary, as described in Refs. 7 and 16:

$$
h(x)=\bar{h}-h^{\prime} \cos \left(\frac{2 \pi x}{L}\right)
$$




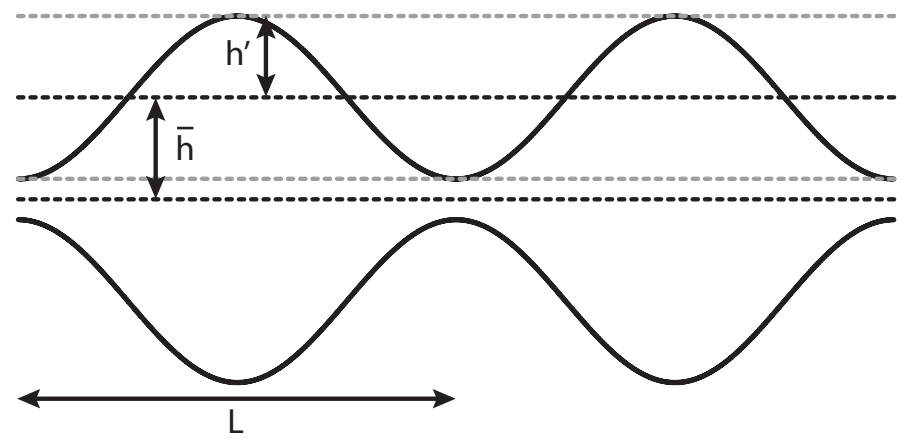

FIG. 1. Geometry of two consecutive unit cells. $\bar{h}$ is the average half-aperture, $h^{\prime}$ define the aperture fluctuation, and $L$ is the length of the unit cell, or wavelength of the sinusoidally varying channel.

where $h$ is the half-aperture at horizontal position $x, \bar{h}$ is the average half-aperture, $h$ ' is the amplitude of the aperture fluctuation, and $L$ is the length of the "unit" cell (see Fig. 1). The fluid flows from left to right.

This geometry can be fully characterized by two dimensionless numbers, namely, the aspect ratio of the cell $\epsilon$,

$$
\epsilon=\frac{2 \bar{h}}{L},
$$

and the relative amplitude of the aperture fluctuations, $a$,

$$
a=\frac{h^{\prime}}{2 \bar{h}} .
$$

When $a$ equals its maximum value of $1 / 2$, the channel is closed and pores are disconnected from the network. When $a$ goes to 0 , the channel is smooth and goes to the parallel plate geometry.

While this is obviously a simplified model for a real porous medium, Edwards et al. ${ }^{11}$ illustrated that it is likely relevant for representing flow and transport in a cylindrically packed porous medium. It has also sometimes been considered as an idealized model for the geometry of a geological fracture, ${ }^{46}$ although realistic fracture geometries are known to be even more complicated. ${ }^{47} \mathrm{~A}$ recent study demonstrated that many of the qualitative and quantitative features regarding velocity distributions and influence on solute transport of a more complex porous medium are well represented by such a simple geometry. ${ }^{48}$

The channel half mean aperture $\bar{h}$, the mean fluid velocity $\bar{u}$, defined in two dimensions as the ratio of the constant volumetric flow rate to $\bar{h}$, and the fluid cinematic viscosity $v$ control the Reynolds number

$$
R e=\frac{2 \bar{h} \bar{u}}{v} .
$$

On a typical pore scale, Reynolds numbers are usually small, ${ }^{16,49}$ of the order of $10^{-4}$ to $10^{-1}$. For such Reynolds numbers, flow is described by the linear Stokes equation. For a slowly varying boundary, i.e., $\epsilon \ll 1$ and small Reynolds number, Kitanidis and Dykaar ${ }^{16}$ derived an analytical solution for the flow velocity using a perturbation expansion in $\epsilon$. However, at larger Reynolds numbers when inertial terms cannot be neglected, this semi-analytical approach is no longer valid; to the best of our knowledge no obvious analytical approach exists to solving the nonlinear governing Navier-Stokes equations.

\section{B. Basic equations and numerical simulations}

\section{Flow}

Direct numerical simulations of the steady state flow through the geometry described in Sec. II A were conducted. The flow is assumed to be incompressible. The conservation of mass 
therefore reads as

$$
\nabla \cdot \boldsymbol{u}=0
$$

where $\boldsymbol{u}$ is the velocity field. The conservation of momentum equation is expressed by the Navier-Stokes equation

$$
\rho\left(\frac{\partial \boldsymbol{u}}{\partial t}+(\boldsymbol{u} \cdot \nabla) \boldsymbol{u}\right)=\rho \boldsymbol{g}-\nabla p+\eta \nabla^{2} \boldsymbol{u},
$$

where $\rho$ is the density of the fluid, $\boldsymbol{g}$ is the gravity field, and $\eta$ is the dynamic viscosity of the fluid.

The system of joint equations (5) and (6) are solved using a finite element method. The finite element numerical simulation is conducted with the commercially available software COMSOL MULTIPHYSICS, in two dimensions and using Lagrange-quadratic elements. The flow is solved on a mesh consisting of triangular elements (up to 100 000) with a maximum side length fixed to $L / 280$. At larger scales, there is no anisotropy induced by the meshing. The solver computes the transient flow iteratively until a stationary solution has been obtained.

The left and right in- and outflow boundaries are treated as periodic, that is, the flow velocity $\boldsymbol{u}$ across the cross section is the same at the inlet and at the outlet. A mean flow is imposed on the inlet boundary. The outlet is set to a constant pressure, which allows the solver to adjust the pressure in the geometry to suit the globally imposed volumetric flow. The details of the numerical method for flow, applied to a different but similar geometry, is described in detail in Ref. 24.

\section{Transport}

We neglect any possible density-driven retroaction of transport on flow, i.e., we assume that the presence of the solute plays a negligible role in changing the density of the fluid. The transport problem is therefore treated once the flow field has been solved for. It is solved numerically using Lagrangian particle tracking random walk simulations based on the Langevin equation. This approach is chosen for two reasons: (i) because of the periodic domain considered here, one does not have to a priori impose the size of the domain and one can allow transport to occur over as a large a distance/computational domain as desired and (ii) because Lagrangian methods do not suffer from problems associated with numerical diffusion in the same way that Eulerian methods can; as we are trying to quantify a dispersive effect we wish to minimize uncertainties on the results as much as possible. The initial condition that we choose is a line uniformly distributed across the width of the channel, i.e.,

$$
c(x, z)=\delta(x)
$$

In discrete time, the equation of motion of the $n$th solute particle, located at position $\boldsymbol{x}^{\prime}$ at initial time, is given by the Langevin equation

$$
\begin{aligned}
& x^{(n)}\left(t+\Delta t \mid \boldsymbol{x}^{\prime}\right)=y^{(n)}\left(t \mid \boldsymbol{x}^{\prime}\right)+u^{(n)}\left(t \mid \boldsymbol{x}^{\prime}\right) \Delta t+\eta_{1} \sqrt{2 D_{\mathrm{m}} \Delta t}, \\
& y^{(n)}\left(t+\Delta t \mid \boldsymbol{x}^{\prime}\right)=y^{(n)}\left(t \mid \boldsymbol{x}^{\prime}\right)+v^{(n)}\left(t \mid \boldsymbol{x}^{\prime}\right) \Delta t+\eta_{2} \sqrt{2 D_{\mathrm{m}} \Delta t}
\end{aligned}
$$

where $\boldsymbol{x}^{(n)}=\left(x^{(n)}, y^{(n)}\right)$ denotes the position of the particle and $\boldsymbol{u}^{(n)}=\left(u^{(n)}, v^{(n)}\right)$ its velocity. The $\eta_{i}(i=1, \ldots, d)$ are independently distributed Gaussian random variables with zero mean and unit variance. This Langevin equation is equivalent to the Fokker-Planck equation; it is identical to the advection-diffusion equation, which describes the time evolution of the solute concentration field inside the system. Solid boundaries are modeled as elastic reflection boundaries in order to account for their impermeability.

The mean half aperture $\bar{h}$, the mean fluid velocity $\bar{u}$, and molecular diffusion coefficient $D_{\mathrm{m}}$ control the Péclet number

$$
P e=\frac{2 \bar{h} \bar{u}}{D_{\mathrm{m}}}
$$


The average position of the solute plume is that of its center of mass

$$
x_{\mathrm{G}}(t)=\frac{1}{N} \sum_{n=1}^{N}\left[x^{(n)}(t)\right] .
$$

The velocity of the center of mass is the mean horizontal velocity of the solute plume. We normalize it by the mean advection velocity

$$
\tilde{u}_{\mathrm{G}}(t)=\frac{1}{\bar{u}} \frac{d x_{\mathrm{G}}}{d t}
$$

The cross-sectionally integrated mass is

$$
M(x, t)=\int_{-h(x)}^{h(x)} C(x, y, t) d y,
$$

resulting in a mean cross-sectional concentration $\bar{C}(x, t)$ with the form

$$
\bar{C}(x, t)=\frac{1}{2 h(x)} M(x, t) .
$$

We quantify longitudinal dispersion in this system from calculations of the horizontal spatial moments of the plume as it evolves in time. The $i$ th local moment is given by averaging over the $i$ th power of the positions of all $N$ simulated particles originating from a single $\mathbf{x}^{\prime}$,

$$
\mu^{(i)}\left(t \mid \mathbf{x}^{\prime}\right)=\lim _{J \rightarrow \infty} \frac{1}{N} \sum_{j=1}^{N}\left[x^{(j)}(t)\right]^{i}
$$

The global moments are obtained by summation over all initial positions $\mathbf{x}^{\prime}$,

$$
m^{(i)}(t)=\lim _{M \rightarrow \infty} \frac{1}{M} \sum_{m=1}^{M} \mu^{(i)}\left(t \mid \mathbf{x}^{(m)}\right) .
$$

The apparent dispersion coefficient is then given by

$$
D_{\mathrm{a}}(t)=\frac{1}{2} \frac{d}{d t}\left[m^{(2)}(t)-m^{(1)}(t)^{2}\right] .
$$

When particles have had sufficient time to sample all the flow lines by diffusion, the asymptotic apparent dispersion coefficient is typically attained. It is defined as

$$
D_{\mathrm{a}}^{\infty}=\lim _{t \rightarrow \infty} D_{\mathrm{a}}(t)
$$

The transport process can then be considered a one-dimensional longitudinal advection-diffusion process with an effective diffusion coefficient equal to $D_{\mathrm{a}}^{\infty}$.

The Taylor-Aris dispersion occurring in a channel of uniform aperture is a well-known limit case for the configuration studied here. In this study, we wish to compare only the term induced by the coupling of diffusion and advection processes and remove the longitudinal diffusion term alone. We do so by subtracting $D_{\mathrm{m}}$ from both our dispersion coefficients and the Taylor-Aris dispersion coefficient $D_{\text {T.A. }}{ }^{1,17}$ thereby defining a normalized dispersion coefficient as

$$
\tilde{D}_{\mathrm{a}}(t)=\frac{D_{\mathrm{a}}(t)-D_{\mathrm{m}}}{D_{\text {T.A. }}-D_{\mathrm{m}}},
$$

which for the particular asymptotic dispersion coefficient is

$$
\tilde{D}_{\mathrm{a}}^{\infty}=\frac{D_{\mathrm{a}}^{\infty}-D_{\mathrm{m}}}{D_{\mathrm{T} . \mathrm{A} .}-D_{\mathrm{m}}} .
$$


Here, $D_{\text {T.A. }}$ is defined for a parallel plate fracture with identical mean aperture and is given by (Wooding ${ }^{18}$ )

$$
D_{\text {T.A. }}=D_{\mathrm{m}}+\frac{2}{105} \frac{(\bar{u} \bar{h})^{2}}{D_{\mathrm{m}}}
$$

In terms of Peclet number it can be written as

$$
D_{\text {T.A. }}=D_{\mathrm{m}}\left[1+\frac{2}{105} P e^{2}\right] .
$$

The simulations release $N$ particles distributed evenly along the cross section of the channel at horizontal position $x=0$. This means that $\boldsymbol{x}^{\prime}$ in Eqs. (14) and (15) is $(0, y)$, the vertical position $y$ being distributed uniformly between $-\bar{h}+h^{\prime}$ and $\bar{h}-h^{\prime}$. For all the time dependent studies, we normalize time by $\tau$, a characteristic advection time defined as the time needed for a particle moving at the mean velocity $\bar{u}$ to cross a single unit cell

$$
\tau=\frac{t \bar{u}}{L}
$$

Additionally, for convenience, $\bar{u}$ is set to 1 for all the simulations and the total mass injected is equal to 1 .

\section{RESULTS}

In Secs. III A and III B below we first describe the observed flow and transport, respectively, qualitatively. We then study the longitudinal effective dispersion coefficient quantitatively.

\section{A. Flow phenomenology}

The presence of recirculation zones depends on the geometry $(\epsilon$ and $a)$ and on the Reynolds number. Examples are given in Fig. 2. At very small Reynolds numbers, Stokes flow conditions are
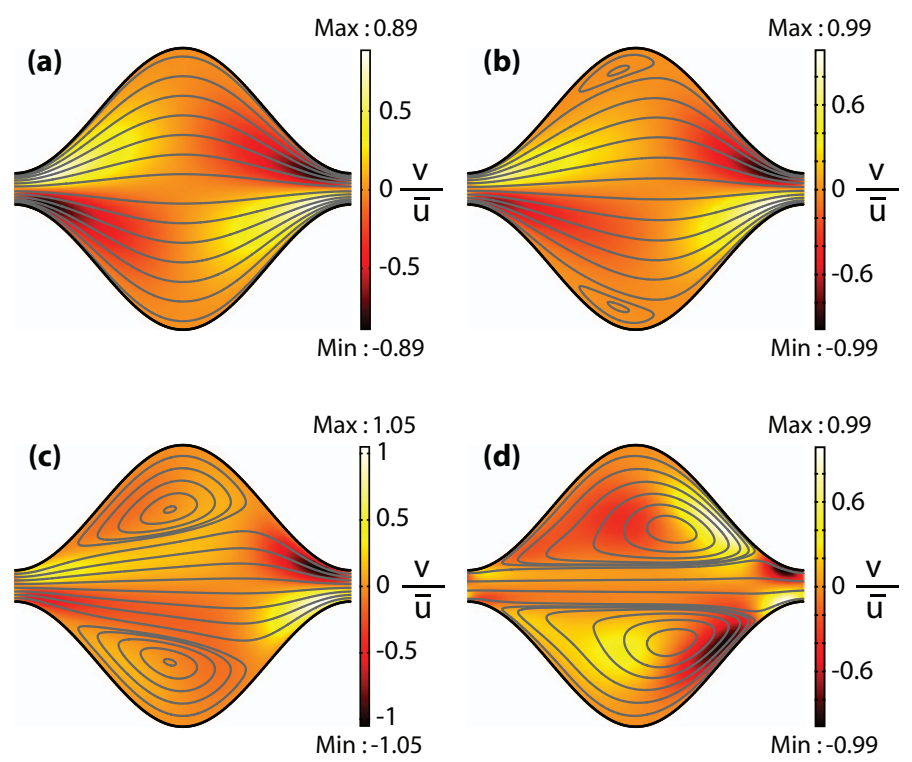

FIG. 2. The shade/color maps show the vertical component of the velocity field, $v$, normalized by the mean horizontal velocity $\bar{u}$. Flow lines are superimposed. The cell geometry is $(\epsilon=0.47, a=0.4)$, and four Reynolds numbers are considered: In (a), $R e=0.1$ and the ratio between the volume of the recirculation zone and the volume of the cell $\phi$ is $0 \%$; in (b), $R e=10$ and $\phi=10 \%$; in (c), $R e=20$ and $\phi=47 \%$; in (d), $R e=100$ and $\phi=75 \%$. Fluid flows from left to right. 

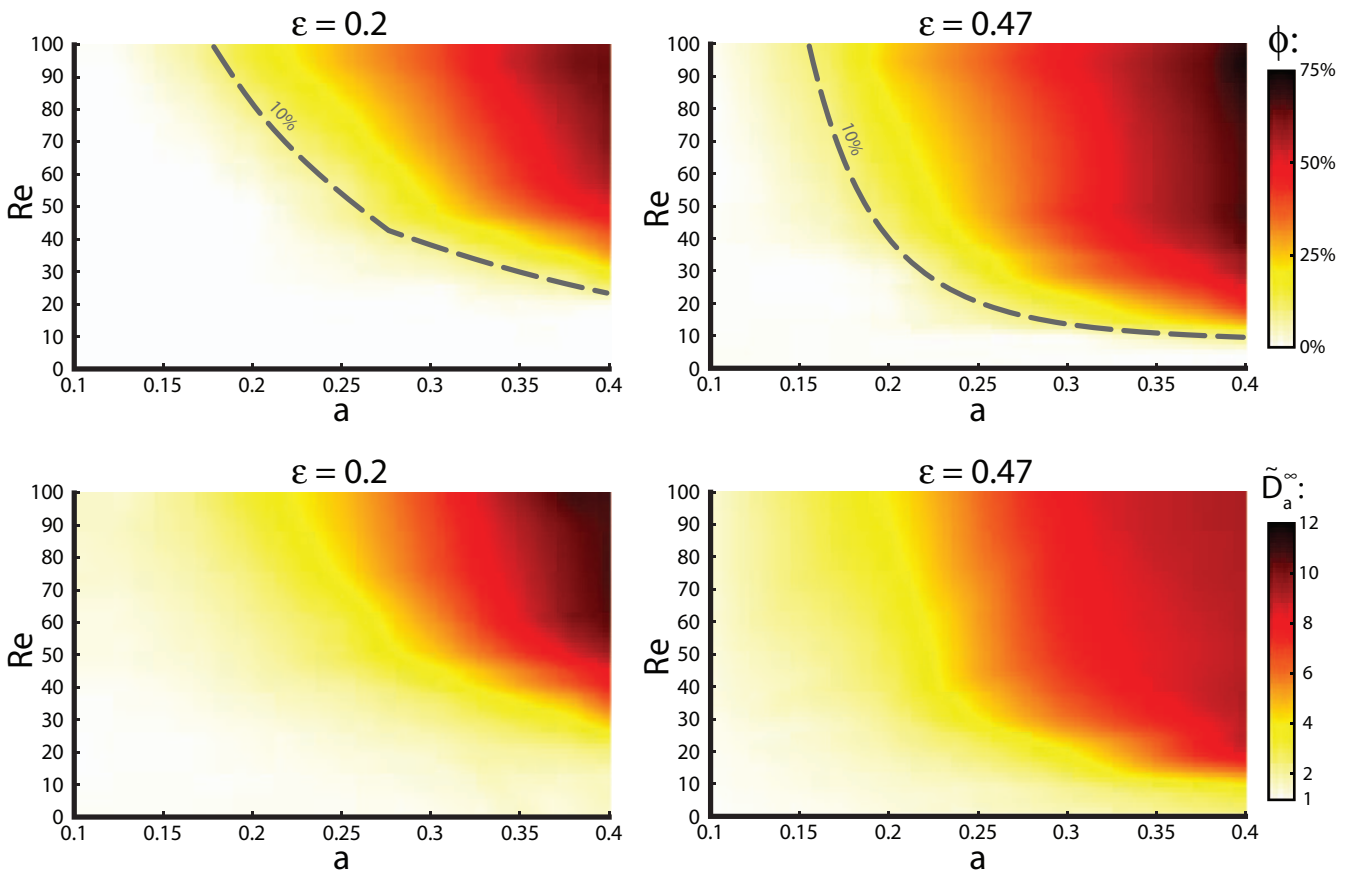

FIG. 3. Volume of the recirculation zone (first row) and normalized asymptotic dispersion coefficient (second row) as a function of the Reynolds number and the relative aperture fluctuation $a$, for two values of the cell aspect ratio $\epsilon$ and $P e=100$. The dashed line represents the $10 \%$ threshold below which the transport is slightly to not impacted by the presence of a recirculation zone. The data are interpolated using a trilinear interpolation.

fulfilled and the flow is reversible when changing the direction of time, which results in its geometry being symmetric with respect to the vertical line $x=L / 2$, as shown in Fig. 2(a). In this figure, no recirculation zones are to be seen: each streamline is homothetic to one of the walls (Fig. 2(a)); note that this homothecy between the walls and streamlines is not enforced by the flow equations and thus not always true: when $\epsilon$ and $a$ are both sufficiently large, recirculation zones are visible even in Stokes flow conditions. ${ }^{7,16}$ At larger Re values inertial effects become evident and the flow lines and vertical velocity maps become asymmetric with respect to the vertical line $x=L / 2$ (Fig. 2(b)), showing that the flow is no longer reversible. The recirculation zones appear in the widest part of the cell in Fig. 2(c), and their size grows monotonically with $\operatorname{Re}$ (see Fig. 2(c) for which $\epsilon=0.47$, $a=0.4$, and $R e=20$ ). The growth is asymmetric but ultimately leads to a flow shape similar to that seen with a single fracture with perpendicular lateral dead ends such as the geometry studied by Lucas. $^{33}$

We define the volume fraction of the recirculation zone $\phi$ as the ratio of the volume of the recirculation zone to that of the cell, $V_{\mathrm{RZ}} / V$. It provides a measure of what fraction of the pore space corresponds to a flow that is bounded in the longitudinal direction and, therefore, will not contribute to advecting particles from one unit cell to the next one.

$\phi$ is a function of the geometry and the Reynolds number (see Fig. 3). Maps of $\phi$ as a function of the relative aperture fluctuation $a$ and the Reynolds number are shown in the top row of Fig. 3 for two values of the cell aspect ratio $\epsilon$. The isoline $\phi=10 \%$ is chosen as the threshold at which we consider that the presence of these recirculation zones begins to have a significant impact on asymptotic transport (i.e., on the asymptotic dispersion coefficient). Depending on the geometry, the $10 \%$ limit can be reached at a quite low Reynolds numbers. For example, for $a=0.25$, i.e., when the aperture at the $x$ position at which the fracture is the widest is equal to 3 times the aperture in the channel throat, Reynolds number values as low as 20 are sufficient for recirculation zones to significantly impact transport. But we shall see in Sec. III D that for $a=0.166$ (i.e., the $\max / \mathrm{min}$ aperture ratio being 2), transport is unaffected by recirculation zones for $R e \lesssim 100$. 


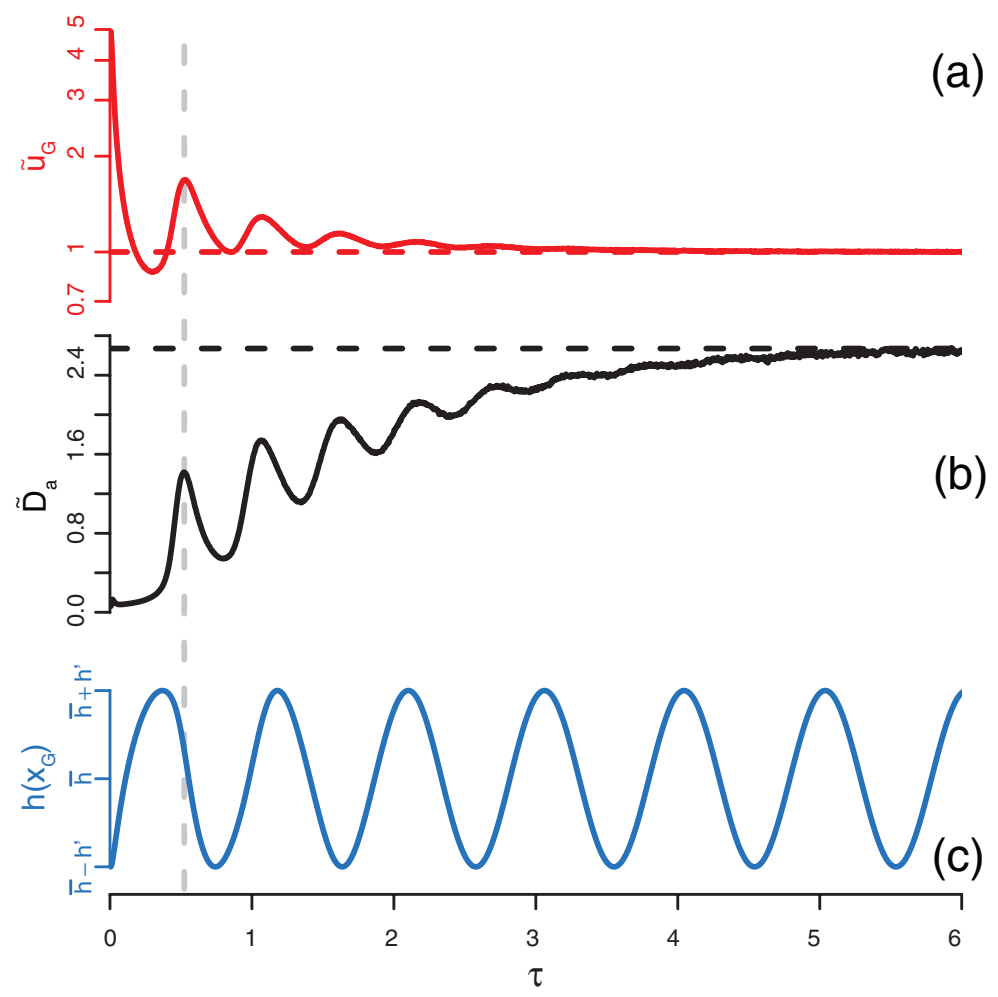

FIG. 4. Evolution of (a) the normalized mean horizontal velocity $\tilde{u}_{\mathrm{G}}$, (b) the normalized dispersion coefficient $\tilde{D}_{\mathrm{a}}$, and (c) the aperture at the position of the center of mass, $h\left(x_{\mathrm{G}}\right)$, as a function of the reduced time $\tau=t \bar{u} / L$. The geometry is defined by $\epsilon=0.47$ and $a=0.4$, flow and transport by $P e=100$ and $R e=1$.

\section{B. Transport phenomenology}

Figure 4 shows a representative analysis of the observables for the transport in a case where $P e=100, R e=1, \epsilon=0.47$, and $a=0.4$. The flow configuration is very similar to the one shown in Fig. 2(a), without any recirculation zone. We expect that once all the particles have experienced all velocities in the domain, the horizontal velocity of the center of mass should converge to the mean flow velocity. This behavior is confirmed in Fig. 4(a). The normalized dispersion coefficient $\tilde{D}_{\text {a }}$ also tends to an asymptotic value, which is larger than 1 . It means that the effective diffusion coefficient value is larger than the one predicted for the uniform aperture case of identical mean aperture.

The early time oscillations of the dispersion coefficient are directly related to the oscillation of the velocity of the center of mass due to the spatial variability in aperture, flow incompressibility, and imposed constant flow rate. The shape of the time evolution of the solute mean velocity is directly related to the geometry parameters ( $\epsilon$ and $a$ ), Reynolds number $(R e)$ and Péclet number $(P e)$. When $a$ increases, the velocity difference between the widest and thinnest zone becomes larger and the oscillation amplitudes increase. When $\epsilon$ increases, the length of the cells is smaller and thus the frequency of the oscillation increases. The effect of an increase of $R e$ alone is a widening of the recirculation zones such that the longitudinal flow appears similar to the parallel plate case (the oscillation amplitude decreasing dramatically) but with a smaller effective mean aperture. Consequently, the velocity of the plume center of mass also increases as most of the solute is in the mainstream (that is outside of the recirculation zone), leading to a higher oscillation frequency. When $P e$ decreases, the higher diffusion coefficient contributes to reaching the asymptotic regime sooner, which means that the oscillations are dampened more quickly.

The first peak of the dispersion coefficient in Fig. 4 occurs at $\tau \approx 0.5$ (shown with a dashed gray line) and nearly coincides with the first peak of the horizontal velocity. A snapshot of the spatial solute distribution at this moment is shown in Fig. 5. A significant part of the solute mass is in the 

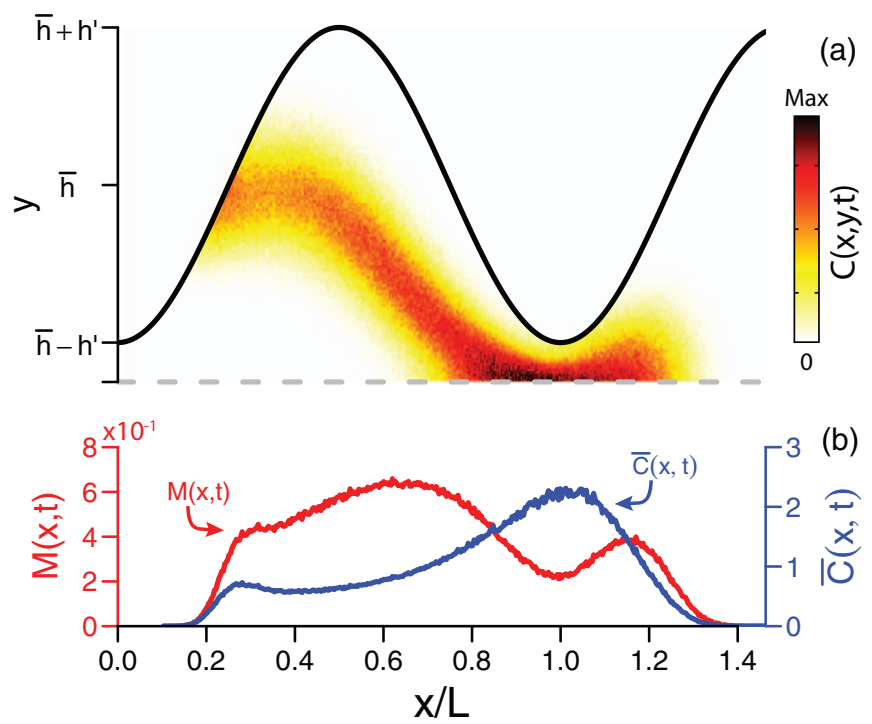

FIG. 5. Snapshot of (a) the concentration field and (b) the vertically summed mass $M(x, t)$ and vertically averaged concentration $\bar{C}(x, t)$ at the first peak time in the longitudinal dispersion coefficient evolution shown by the gray dashed line in Fig. 4 (i.e., at $\tau \approx 0.5$ ). The geometry is defined by $\epsilon=0.47$ and $a=0.4$, flow and transport by $P e=100$ and $R e=1$.

cell throat where the velocity is at a maximum, while the rest of the solute is in a relatively slow zone. In this configuration, the plume is highly stretched, leading to a large dispersion coefficient value.

Figures 6 and 7 provide a comparison of the spatial distribution of the concentration at a time $\tau \approx 1.25$ for two opposite cases; a highly diffusive one with smaller Péclet number $(P e=50)$ (Fig. 6) and a highly advective one with large Péclet number $(P e=500)$ (Fig. 7). The Reynolds number is high in both cases $(R e=200)$, with fully developed recirculation zones, as seen in Fig. 2(d). Note that the maximum concentration values are different and so are the color scales. In the first cell, vertically averaged solute concentration is very similar in both cases. In Fig. 7, solute barely enters the recirculation zones and most of the mass is in the center of the cells, moving
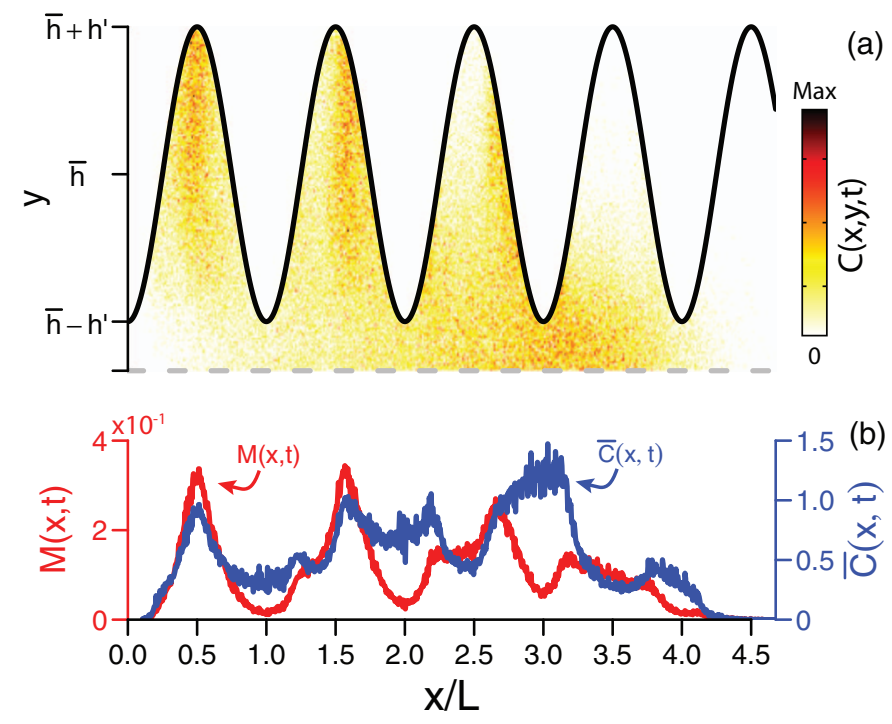

FIG. 6. Snapshot of (a) the concentration field and (b) the vertically summed mass $M(x, t)$ and vertically averaged concentration $\bar{C}(x, t)$ in a geometry with $\epsilon=0.19$ and $a=0.38$ at time $\tau \approx 1.25$. The configuration is highly diffusive $(P e=50$, $R e=200)$. 

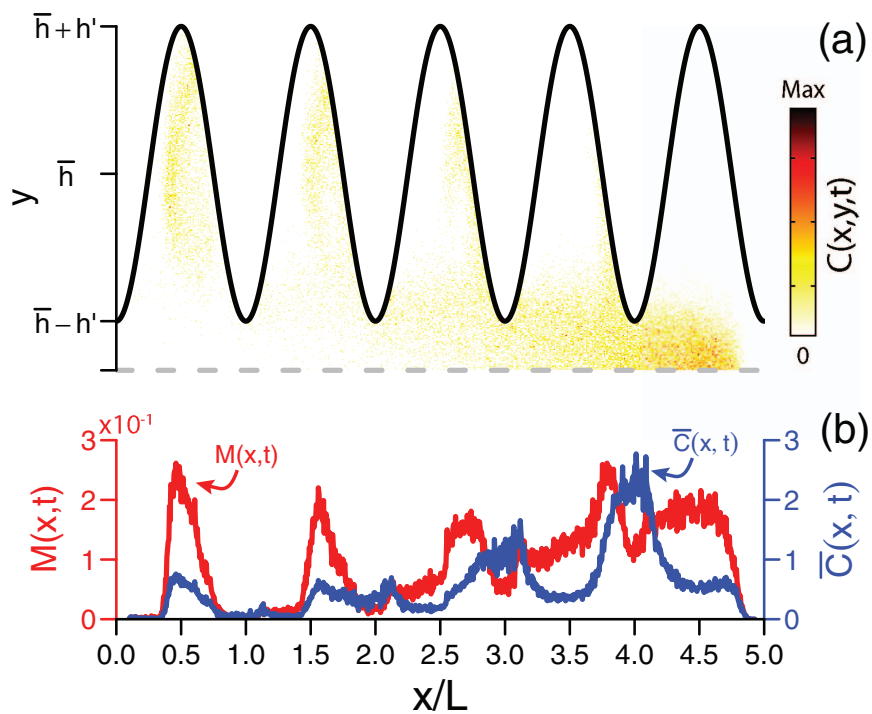

FIG. 7. Snapshot of (a) the concentration field and (b) the vertically summed mass $M(x, t)$ and vertically averaged concentration $\bar{C}(x, t)$ in a geometry with $\epsilon=0.19$ and $a=0.38$ at time $\tau \approx 1.25$. The configuration is highly diffusive $(P e=500$, $R e=200)$.

quickly. Particles can jump by diffusion on to a line on the edge of the recirculation zone and then enter more deeply into the zone by advection. As such, they are observed to be advected deeper into the recirculation zone by the right side of the unit cell. As the diffusion coefficient is small while the probability to enter the recirculation zone is low, the probability to exit it again is also low. On the contrary, in Fig. 6 a significant amount of the total mass has been able to enter the recirculation by diffusion. In Fig. 6(b), the particles are nearly equally distributed in the three first cells and begin to enter the fourth cell. The concentration values are mostly in the range $[0.3,1]$ and three throat zones are not empty. In Fig. 7(b), most of the particles are at the front of the plume, even if a significant amount is trapped in the recirculation zones. The concentration is already very low in the first throats but high at the front of the plume. The concentration values are mostly in the range $[0,2]$.

\section{Time evolution of the apparent dispersion coefficient}

For most of the simulations, as illustrated in Fig. 4 for $\epsilon=0.47$ and $a=0.4$, the dispersion coefficient oscillates in time before reaching an asymptotic value (see Figure 8). These early time oscillations are directly related to the shape of the geometry and to the evolution of the velocity of the center of mass; in particular, the oscillations of the dispersion coefficient and those of $\tilde{u}_{\mathrm{G}}$ seem to be in phase. The oscillations dampen over time, and their oscillation amplitude increases when $R e$ decreases or $a$ increases. The oscillations also tend to dampen more quickly with a lower $P e$, due to more rapid diffusive smearing. The oscillation frequency is directly related to a mean velocity calculated only in the zone outside of the recirculation zones. Thus, it increases when $R e$ or $\epsilon$ increases.

It turns out that, once normalized by a proper $\tilde{u}_{\mathrm{G}}^{\lambda}$ law, the longitudinal dispersion data exhibit next to no fluctuations reminiscent of the sinusoidal boundary conditions (see inset in Fig. 8); additionally, we note that it is well described by a stretched exponential behavior. In other words, the $D_{\text {a }}$ data have excellent fitted a law of the form (Fig. 8),

$$
D_{\mathrm{a}}(\tau)=D_{\mathrm{a}}^{\infty}\left(1-\exp \left[-\left(\frac{\tau}{\tau_{c}}\right)^{\gamma}\right]\right) \tilde{u}^{\lambda},
$$

where $D_{\mathrm{a}}^{\infty}$ is the asymptotic dispersion coefficient, $\tau_{c}$ is a characteristic time, $\tilde{u}_{\mathrm{G}}$ is the rescaled velocity of the solute center of mass as defined by Eq. (11), and $\gamma$ is the exponent inside the stretched 


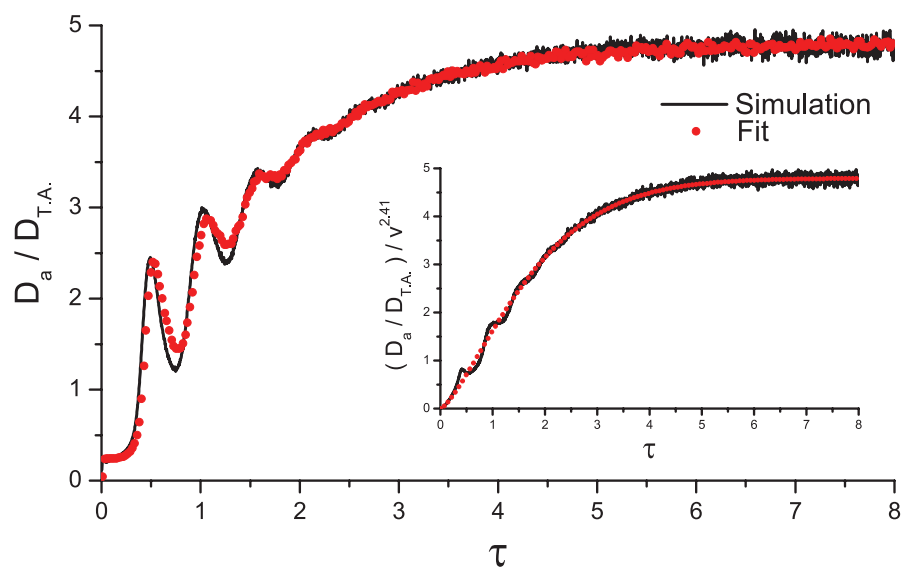

FIG. 8. Time evolution of the normalized dispersion coefficient and fit comparison using Eq. (23). In this case, $P e=50$, $R e=10, \epsilon=0.47$, and $a=0.4$. The characteristic time to the asymptote is $\tau_{c}=4.10$, the asymptotic dispersion coefficient is $D_{\mathrm{a}}^{\infty} / D_{\text {T.A. }}=4.80$ and the power coefficients are $\gamma=1.36$ and $\lambda=2.41$.

exponential. The fitting parameters $D_{\mathrm{a}}^{\infty}, \tau_{c}, \gamma$, and $\lambda$ correspond to distinct geometric properties of the curve, and are therefore obtained with only a small degree of uncertainty.

In addition to properly describing the whole time evolution of the longitudinal dispersion, the fit provides us with a robust estimate of the asymptotic value for the dispersion coefficient. We will focus on this observable hereafter.

\section{Asymptotic dispersion coefficient as a function of flow and geometry parameters}

\section{Dependence on the Reynolds and Péclet numbers}

For each geometry, the asymptotic dispersion coefficient $\tilde{D}_{\mathrm{a}}^{\infty}$ is computed from the fit of the apparent longitudinal dispersion coefficient with time. In Fig. 9(a), we show how it varies as a function of $R e$ and $P e$. Under conditions of lower Reynolds number, $\tilde{D}_{\mathrm{a}}^{\infty}$ is controlled by the Péclet number. When the Reynolds number increases, $\tilde{D}_{\mathrm{a}}^{\infty}$ increases and then reaches an asymptotic value at a large Reynolds number. Indeed, when a sufficiently high Reynolds number is reached, the recirculation zone ceases to grow further (or at least grows very slowly). The numerical model is only valid when the flow remains stationary, which requires that no transition to turbulence occurs in any part of the system, so we never perform simulation with Reynolds greater than 250 . With this limit, we can observe the plateau for only a few geometries that have large values of $a$. For most of them, only the low Reynolds plateau and the beginning of the dispersion coefficient increase are observed. Note that the inset in Fig. 9(a) shows the same data as a function of the Péclet number, for the various Reynolds numbers investigated; obviously the behavior as a function of $P e$ is a power law whose exponent hardly depends on $R e$.

Our goal is to find an empirical relation for $\tilde{D}_{\mathrm{a}}^{\infty}$ as a function of $R e$ and $P e$. For a given geometry and for $P e>50$, all the curves of $\tilde{D}_{\mathrm{a}}^{\infty}$ as a function of $R e$ collapse in one by applying a scaling coefficient $\kappa$ (Fig. 9(b)). For a given geometry, this scaling coefficient only depends on Pe. As shown in the inset of Fig. 9(b), $\kappa$ scales as a power law of the Péclet

$$
\kappa=\alpha P e^{\beta} .
$$

This scaling simply derivates from the power law behavior shown in the inset of Fig. 9(a).

\section{Global scaling}

The asymptotic dispersion coefficient values can be described by

$$
\tilde{D}_{\mathrm{a}}^{\infty}(P e, R e)=\left[\alpha P e^{\beta}\right] f(R e),
$$



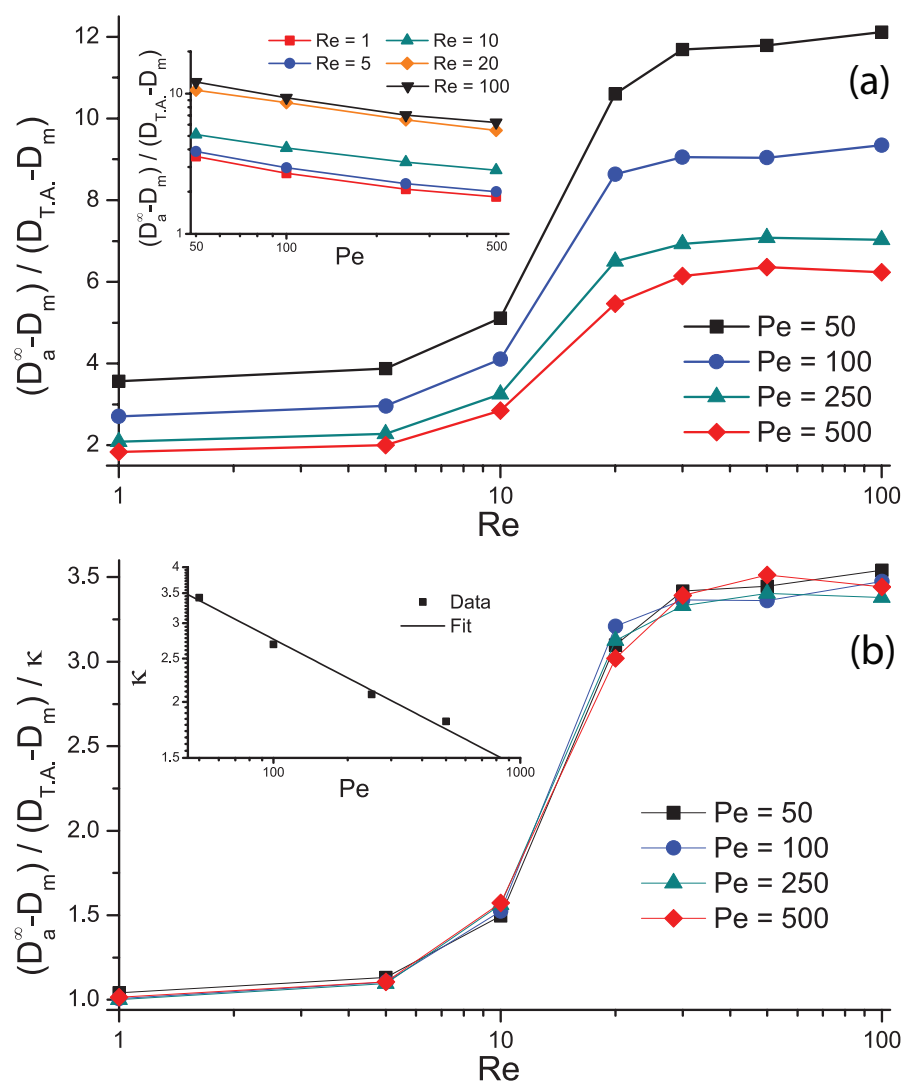

FIG. 9. (a) Normalized asymptotic dispersion coefficient as a function of $R e$ for several $P e$ values; the inset shows the same data plotted as a function of the Péclet, for all values of $R e$. (b) shows the same data once scaled and the inset in (b) shows the fit of the scaling coefficient $\kappa$ as a function of $P e$. The geometry parameters are $\epsilon=0.47$ and $a=0.4$.

that is,

$$
D_{\mathrm{a}}^{\infty}(P e, R e)=D_{\mathrm{m}}\left[1+\frac{2}{105}\left(\alpha P e^{\beta+2}\right) f(R e)\right],
$$

where $\alpha$ and $\beta$ are coefficients that only depend on the geometry and $f(R e)$ is a function of $R e$ with small and large asymptotic values at small and large Re, respectively. A linear combination of the error function and the $y=1$ constant function seems suitable. As the second plateau is never reached for most of the geometries, it is difficult to infer a precise functional shape for the fit.

Equation (26) can be compared directly with Eq. (21). The asymptotic dispersion coefficient description and the Taylor-Aris dispersion coefficient share similarities. The latter classic form is complemented with a correction $\beta$ to the power of $P e$ and with an additional factor that only depends on the Reynolds number.

Figure 10 shows the relation between the parameters $\alpha$ and $\beta$ and the relative amplitude of the aperture fluctuations, $a$. Several geometries corresponding to the same $a$ but to different cell aspect ratios $\epsilon$ share an identical $\alpha$ : this figure suggests that there is no significant dependence of the prefactor $\alpha$ of Eq. (26) on $\epsilon$. In contrast, $\alpha$ increases monotonically with $a$. Similarly, the exponent parameter $\beta$ appears to depend weakly on $\epsilon$, but exhibits a marked decreasing trend as a function of $a$. When $a$ tends to $0, \alpha$ tends to $1, \beta$ to 0 , and thus $\kappa$ tends to 1 . This is consistent with the fact that very small values of $a$ correspond to geometries that approach the parallel plate geometry, for which $D_{\mathrm{a}}^{\infty}=D_{\text {T.A. }}$. 


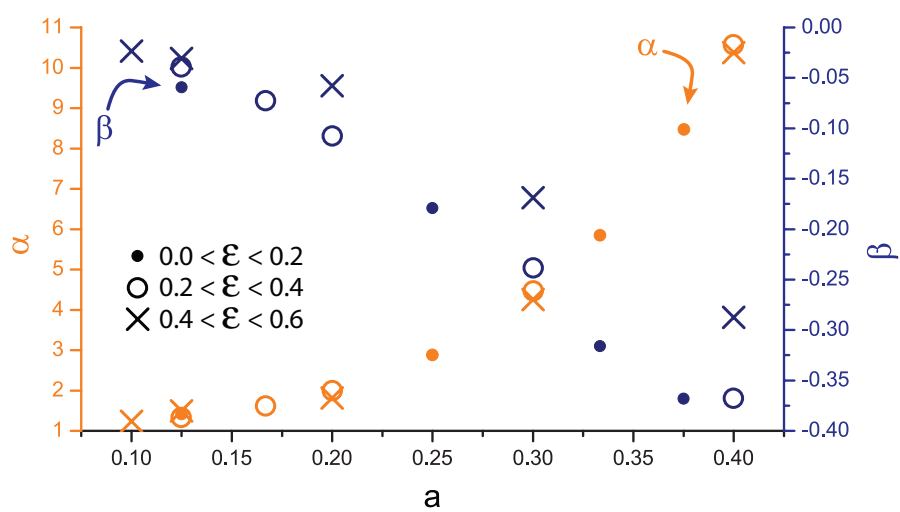

FIG. 10. Fitting parameters $\alpha$ (orange online symbols) and $\beta$ (blue online symbols) as a function of $a$. The three types of symbols denote three different ranges of values for the cell aspect ratio $\epsilon$.

\section{Relation between the size of the recirculation zones and the asymptotic dispersion coefficient}

In the bottom row of Fig. 3, we plot maps of the normalized asymptotic dispersion coefficient as a function of the relative amplitude of the aperture fluctuations, $a$, and the Reynolds number, for the same two values of the cell aspect ratio $\epsilon$ as chosen to illustrate the size of the recirculation zones in the top row of the same figure. These maps of $\tilde{D}_{\mathrm{a}}^{\infty}$ have been obtained by interpolating all available $\tilde{D}_{\mathrm{a}}^{\infty}$ data, measured for various Reynolds numbers and a variety of values of $a$. For example, the curve in Fig. 9(a) for $P e=100$ is a vertical slice of Fig. 3 at $a=0.4$. Accordingly, the topography of the $\tilde{D}_{\mathrm{a}}^{\infty}$ maps in Fig. 3 is consistent with the plots discussed in Sec. III D 1. In particular, Fig. 3 illustrates that when $a$ goes to $0, \tilde{D}_{a}^{\infty}$ goes to 1 , and thus $f(R e)$ to 1 .

A comparison of the two rows of Fig. 3 shows that for a given Péclet number value, the dispersion coefficient evolution is tightly correlated to the volume of the recirculation zones, $\phi$. This is a direct consequence of the phenomenology of transport as discussed in Sec. III B, and of solute trapping in recirculation zones at the back of the solute cloud. In this respect, the asymptotic dispersion is also expected to be related to the variance of the velocity fluctuations, and indeed maps (not shown here) of this variance as a function of $a$ and the Reynolds appear very similar to those of $\phi$ shown in the first row of Fig. 3.

\section{E. Characteristic time to reach the asymptotic regime as a function of flow and geometry parameters}

For each geometry, the characteristic time $\tau_{c}$ necessary to reach the asymptotic regime is obtained from the fit of the time evolution of the apparent longitudinal dispersion coefficient. Figure 11 shows its evolution as a function of $R e$ for various $P e$ values. Figure 11(a) is obtained with a geometry defined by $\epsilon=0.47$ and $a=0.4$, while Fig. 11(b) corresponds to a geometry defined by $\epsilon=0.19$ and $a=0.38$. In others words, the values of $a$ are similar in the two configurations, but the $\epsilon$ values differ by a factor of two. The characteristic time is likely related to the minimum duration needed for a particle to experience the whole cross-sectional profile of velocities by diffusion, and therefore controlled both by the actual value of the diffusion coefficient and by the characteristic transverse distance particles have to cover by diffusion. This latter aspect is confirmed in Fig. 11. For a given $P e$ value, the asymptotic regime is clearly reached sooner when the cell aspect ratio $\epsilon$ is smaller ((b) case). Also, for a given geometry, the higher the Péclet number, the shorter the characteristic time, reflecting a slower sampling of the velocity heterogeneity as diffusion is weaker.

At intermediate $R e$ values (i.e., when the recirculation zones are growing), the trapping of particles delays the asymptotic regime. The curves are shifted toward larger $R e$ values as the Péclet number decreases: when $P e$ is small, the recirculation zone has to grow significantly (and 

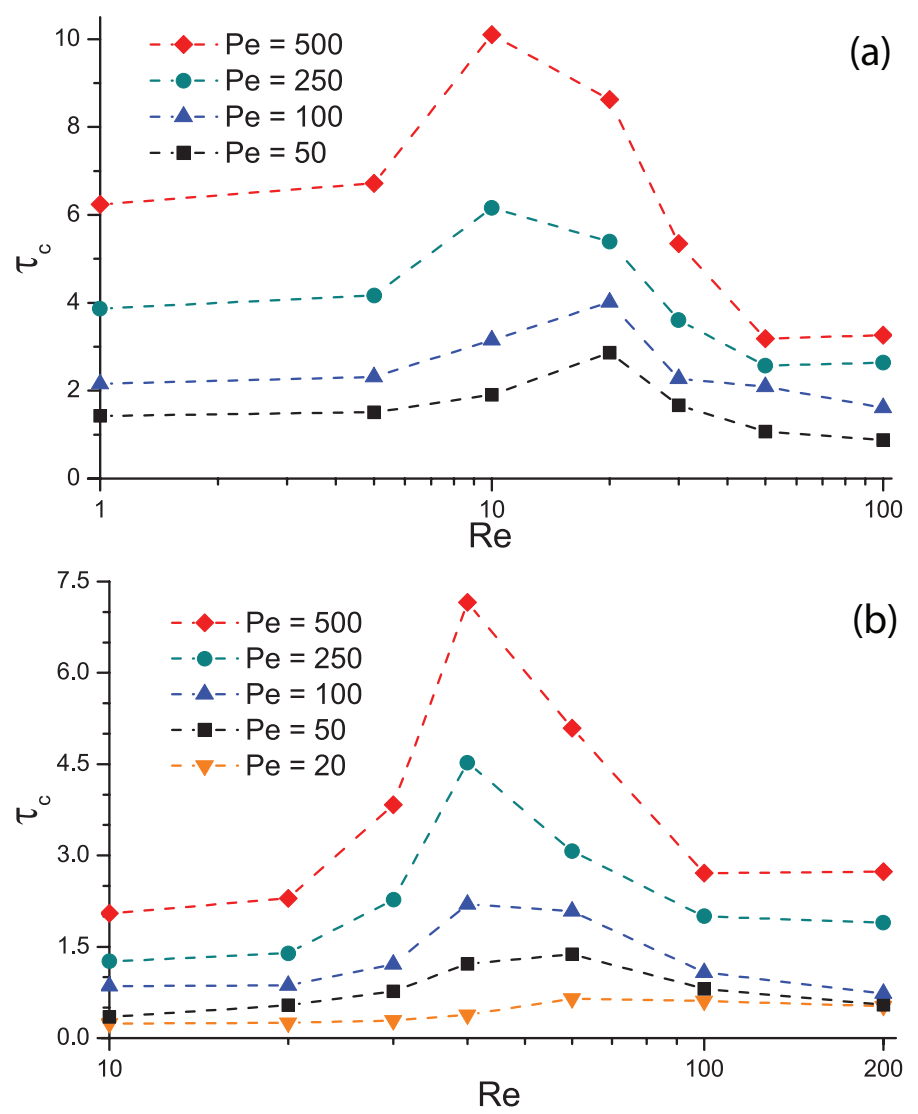

FIG. 11. Characteristic time of the asymptotic regime as a function of $R e$ for several $P e$ values. In (a), geometry parameters are $\epsilon=0.47$ and $a=0.4$; in (b), geometry parameters are $\epsilon=0.19$ and $a=0.38$.

thus, the Re must increase significantly) before the characteristic time rises. The growth of the recirculation zones (and thus the thinning of the mainstream) induces rapid homogenization in the central zone. This phenomenon tends to decrease the characteristic time against the delaying effect of trapping. Thus, trapping in the recirculation zones and faster mixing in the central zone compete in controlling the characteristic time $\tau_{c}$. Depending on the geometry, the Péclet number and the Reynolds number, one of those two phenomena dominates. When the value of $\epsilon$ is large (Fig. 11(a)), the asymptotic regime is reached faster for larger $R e$ conditions than for lower $R e$ conditions because of the enhanced mixing in the mainstream, which dominates over trapping. On the contrary, when $\epsilon$ is smaller (Fig. 11(b)), trapping is predominant and the characteristic time $\tau_{c}$ is lower under large $R e$ conditions than under low $R e$ conditions.

\section{F. Breakthrough curves}

Sample breakthrough curves are given in Fig. 12. The concentration is measured at position $x=50 L$, well after the dispersion coefficient has reached its asymptotic value.

The first arrival time is controlled predominantly by the advection and diffusion of a particle traveling at or close to the center of the cell where the longitudinal velocities are largest. As the recirculation zones grow, the bulk of the longitudinal flow occurs in an increasingly smaller volume of the cell, thus resulting in larger maximum velocities. Thus, the larger the Reynolds number, the smaller the time needed for the fastest particles to travel through the cells.

Lower $P e$ values lead to smaller asymptotic dispersion coefficient values, which manifests itself as higher, but narrower peaks in the breakthrough curves. For the parallel plates geometry case, the asymptotic dispersion coefficient is smallest. 


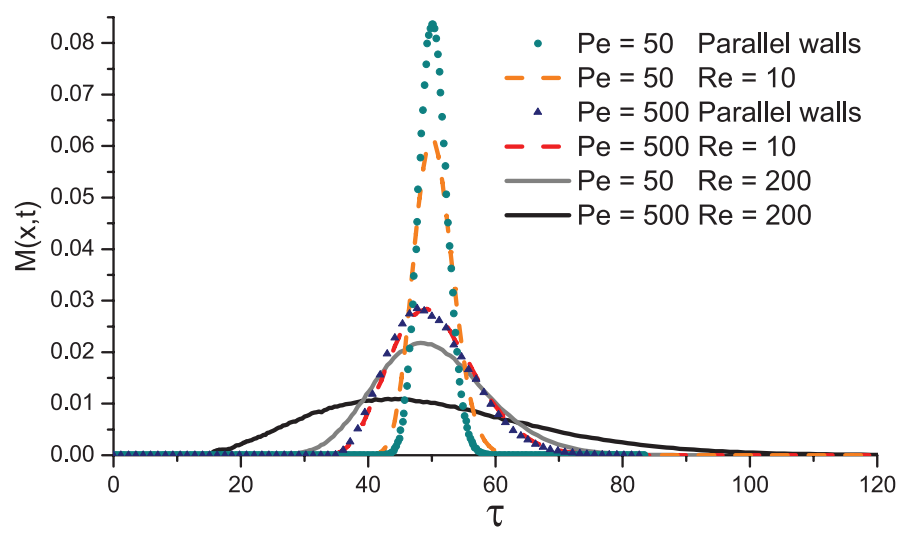

FIG. 12. Breakthrough curves after 50 unit cells have been travelled (i.e., at $x=50 L$ ), for configurations of low and high diffusion $(P e=50$ and $P e=500)$ and for low and high Reynolds numbers $(R e=10$ and $R e=200)$ in a geometry with $\epsilon=0.19$ and $a=0.38$. Additionally, two breakthrough curves for a parallel plate geometry with the same mean aperture (i.e., $a=0$ ) at $P e=50$ and $P e=500$ are shown.

\section{CONCLUSION}

We have studied numerically the impact of inertial effects on flow and transport in channels of periodically varying aperture. In particular, we investigated the conditions under which recirculation zones appear and monitored the volume fraction occupied by these "transport-delaying" zones as a function of the geometrical parameters (i.e., the aspect ratio of a cell and the relative amplitude of the aperture fluctuations). Recirculation zones grow when the aperture fluctuation, the aspect ratio of the cell or the Reynolds number increase. A range of geometry parameters and Reynolds number values exists for which the volume of the recirculation zones is very sensitive to these parameters. For a number of geometries, Reynolds number values as low as 20 are sufficient to create recirculation zones that impact transport significantly. In such geometries, the recirculation zones can be sufficiently large to occupy up to $75 \%$ of the pore volume even when the Reynolds numbers are less than 50. It is important to emphasize that the flow is laminar and not turbulent; recirculation zones that arise from inertial effects are characterized by their asymmetry relative to the longitudinal reflection plane.

As anticipated from the generalized Taylor dispersion theory, the longitudinal apparent dispersion coefficient reaches in time an asymptotic value corresponding to an effective diffusion coefficient for longitudinal transport. The deviation of this asymptotic dispersion coefficient from the equivalent Taylor-Aris value (which corresponds to a parallel plate channel with the same cell aspect ratio and no fluctuation on the boundary) accounts for the impact of the flow complexity on longitudinal transport along the system. We propose an expression for that asymptotic dispersion coefficient (Eq. (26)) that is a generalization of the well-known expression by Taylor and Aris, ${ }^{1,17}$ and that takes molecular diffusion, the Reynolds number, the Péclet number, and the cell geometry into account. The dependence of $D_{\mathrm{a}}^{\infty} / D_{\mathrm{m}}-1$ on the Reynolds number, at least for the range of parameters investigated here, is found to be uncorrelated to the other parameters; that is, its influence appears as a separate factor with a given functional form $f(R e)$. The dependence of $D_{\mathrm{a}}^{\infty} / D_{\mathrm{m}}-1$ on the Péclet is found to deviate from its form in the well-known Taylor-Aris expression, where it scales as $P e^{2}$, to a related, but different power law $P e^{2+\beta}$. The exponent $\beta$ is controlled by the cell geometry. Another effect of the cell geometry is an additional geometry-controlled prefactor $\alpha$ in $D_{\mathrm{a}}^{\infty} / D_{\mathrm{m}}-1$. Both geometric parameters $\alpha$ and $-\beta$ were found not to depend (or only very weakly) on the cell aspect ratio; they are positive increasing functions of the relative amplitude of aperture fluctuations, $a$. One can expect the asymptotic dispersion coefficient to deviate all the more from the Taylor-Aris dispersion coefficient as the relative amplitude of the aperture fluctuations and the Reynolds number increase, and as the Péclet number decreases. For realistic scenarios, the asymptotic dispersion coefficient value could be up to an order of magnitude (or more specifically 12 times) larger than the Taylor-Aris dispersion coefficient. The evolution of the asymptotic disper- 
sion coefficient as a function of the geometry parameters and of the Reynolds number is closely tied to the volume of the recirculation zones, which illustrates in a stunning manner their role in the deviation from the behavior under uniform aperture.

We were also able to infer a functional form for the pre-asymptotic evolution of the dispersion coefficient. Short period initial oscillations of the signal were, in particular, shown to be mostly due to oscillations of the average solute velocity as it passes "pores" and "throats" of the medium. The approach to the asymptotic regime occurs over a characteristic time that is typically much larger than the characteristic advection time (for Peclet number of practical interest $P e>O(1)$ ), and is also impacted by the presence of recirculation zones. The evolution of that characteristic time as a function of the geometrical parameters, the Reynolds number and the Péclet number is controlled by two oppositely competing phenomena. When the recirculation zones are sufficiently large, the trapping of particles inside them delays the establishment of the asymptotic regime. But when the recirculation zone is even larger, the enhanced mixing in the mainstream due to the thinning of that zone leads to faster homogenization and thus a decrease in the characteristic time. Depending on the specific parameter values, one phenomenon dominates and the characteristic time can be either larger or smaller than the one measured under low Reynolds number (Stokes) conditions.

In addition to generalizing the theoretical Taylor-Aris dispersion relation to inertial laminar flows, the current study could also be useful to predict longitudinal dispersion in flows at sufficiently large Reynolds and in systems for which the sinusoidal geometry, albeit not exactly describing the actual geometry, is a decent approximation of the real system. It is, for example, the case for tracer tests under pumping conditions in homogeneous rocks with sufficiently large and well sorted grains.

${ }^{1}$ G. I. Taylor, "Dispersion of soluble matter in solvent flowing slowly through a tube," Proc. R. Soc. London 219(1137), $186(1953)$

${ }^{2}$ L. E. Locascio, "Microfluidic mixing," Anal. Bioanal. Chem. 379(3), 325-327 (2004).

${ }^{3}$ A. Tripathi, O. Bozkurt, and A. Chauhan, "Dispersion in microchannels with temporal temperature variations," Phys. Fluids 17(10), 103607 (2005).

${ }^{4}$ J. H. Forrester and D. F. Young, "Flow through a converging-diverging tube and its implications in occlusive vascular disease - II," J. Biomech. 3(3), 307-316 (1970).

${ }^{5}$ H. Ye, D. B. Das, J. T. Triffitt, and Z. Cui, "Modelling nutrient transport in hollow fibre membrane bioreactors for growing three-dimensional bone tissue," J. Membr. Sci. 272(1-2), 169-178 (2006).

${ }^{6} \mathrm{H}$. Brenner, "Dispersion resulting from flow through spatially periodic porous media," Philos. Trans. R. Soc. London 297(1430), 81-133 (1980).

${ }^{7}$ D. Bolster, M. Dentz, and T. Le Borgne, "Solute dispersion in channels with periodically varying apertures," Phys. Fluids 21(5), 056601 (2009).

${ }^{8}$ D. L. Koch and J. F. Brady, "The symmetry properties of the effective diffusivity tensor in anisotropic porous media," Phys. Fluids 30(3), 642 (1987).

${ }^{9}$ D. Koch and J. Brady, "A non-local description of advection-diffusion with application to dispersion in porous media," J. Fluid Mech. 180(1), 387-403 (1987).

${ }^{10}$ J. Salles, J.-F. Thovert, R. Delannay, L. Prevors, J.-L. Auriault, and P. M. Adler, "Taylor dispersion in porous media: Determination of the dispersion tensor," Phys. Fluids A 5(10), 2348 (1993).

${ }^{11}$ D. A. Edwards, M. Shapiro, H. Brenner, and M. Shapira, "Dispersion of inert solutes in spatially periodic, two-dimensional model porous media," Transp. Porous Media 6(4), 337-358 (1991).

${ }^{12}$ D. Bolster, M. Dentz, and J. Carrera, "Effective two-phase flow in heterogeneous media under temporal pressure fluctuations," Water Resour. Res. 45(5), 1-14, doi:10.1029/2008WR007460 (2009).

${ }^{13}$ G. Dagan and A. Fiori, "The influence of pore-scale dispersion on concentration statistical moments in transport through heterogeneous aquifers," Water Resour. Res. 33(7), 1595, doi:10.1029/97WR00803 (1997).

${ }^{14}$ M. Dentz and J. Carrera, "Mixing and spreading in stratified flow," Phys. Fluids 19(1), 017107 (2007).

${ }^{15}$ A. Fiori, "On the influence of pore-scale dispersion in nonergodic transport in heterogeneous formations," Transp. Porous Media 30(1), 57-73 (1998).

${ }^{16}$ P. K. Kitanidis and B. B. Dykaar, "Stokes flow in a slowly varying two-dimensional periodic pore," Transp. Porous Media 26(1), 89-98 (1997).

${ }^{17}$ R. Aris, "On the dispersion of a solute in a fluid flowing through a tube," Proc. R. Soc. London 235(1200), 67-77 (1956).

${ }^{18}$ R. Wooding, "Instability of a viscous liquid of variable density in a vertical Hele-Shaw cell," J. Fluid Mech. 7(04), 501 (1960).

${ }^{19}$ B. Berkowitz and J. Zhou, "Reactive solute transport in a single fracture," Water Resour. Res. 32(4), 901-913, doi:10.1029/95WR03615 (1996).

${ }^{20}$ V. Zavala-Sanchez, M. Dentz, and X. Sanchez-Vila, "Characterization of mixing and spreading in a bounded stratified medium," Adv. Water Resour. 32(5), 635-648 (2009).

${ }^{21}$ W. N. Gill and R. Sankarasubramanian, "Exact analysis of unsteady convective diffusion," Proc. R. Soc. London 316(1526), 341-350 (1970).

${ }^{22}$ M. Latini and A. Bernoff, "Transient anomalous diffusion in Poiseuille flow,” J. Fluid Mech. 441, 399-411 (2001). 
${ }^{23}$ D. Bolster, F. J. Valdés-Parada, T. Le Borgne, M. Dentz, and J. Carrera, "Mixing in confined stratified aquifers," J. Contam. Hydrol. 120-121, 198-212 (2011).

${ }^{24}$ J. Bouquain, Y. Meheust, and P. Davy, "Horizontal pre-asymptotic solute transport in a plane fracture with significant density contrasts," J. Contam. Hydrol. 120-121, 184-97 (2011).

${ }^{25}$ M. E. Erdogan and P. C. Chatwin, "The effects of curvature and buoyancy on the laminar dispersion of solute in a horizontal tube," J. Fluid Mech. 29, 465-484 (1967).

${ }^{26}$ D. A. Hoagland and R. K. Prud'Homme, "Taylor-aris dispersion arising from flow in a sinusoidal tube," AIChE J. 31(2), 236-244 (1985).

${ }^{27}$ D. M. Tartakovsky and D. Xiu, "Stochastic analysis of transport in tubes with rough walls," J. Comput. Phys. 217(1), 248-259 (2006).

${ }^{28}$ D. Xiu and D. M. Tartakovsky, "Numerical methods for differential equations in random domains," SIAM J. Sci. Comput. (USA) 28(3), 1167 (2006).

${ }^{29}$ L. W. Gelhar, "Stochastic subsurface hydrology from theory to applications," Water Resour. Res. 22(9S), 135S, doi:10.1029/WR022i09Sp0135S (1986).

${ }^{30}$ G. Drazer, H. Auradou, J. Koplik, and J. P. Hulin, "Self-affine fronts in self-affine fractures: Large and small-scale structure," Phys. Rev. Lett. 92(1), 014501 (2004).

${ }^{31}$ S. Rosencrans, "Taylor dispersion in curved channels," SIAM J. Appl. Math. 57(5), 1216 (1997).

${ }^{32}$ B. B. Dykaar and P. K. Kitanidis, "Macrotransport of a biologically reacting solute through porous media," Water Resour. Res. 32(2), 307, doi:10.1029/95WR03241 (1996).

${ }^{33}$ Y. Lucas, M. Panfilov, and M. Bues, "High velocity flow through fractured and porous media: The role of flow nonperiodicity,” Eur. J. Mech. B/Fluids 26(2), 295-303 (2007).

${ }^{34}$ K. Chaudhary, M. B. Cardenas, W. Deng, and P. C. Bennett, "The role of eddies inside pores in the transition from Darcy to Forchheimer flows," Geophys. Res. Lett. 38(24), L24405, doi:10.1029/2011GL050214 (2011).

${ }^{35}$ M. B. Cardenas, D. T. Slottke, R. A. Ketcham, and J. M. Sharp, "Effects of inertia and directionality on flow and transport in a rough asymmetric fracture,” J. Geophys. Res. 114(B6), B06204, doi:10.1029/2009JB006336 (2009).

${ }^{36}$ M. B. Cardenas, "Direct simulation of pore level Fickian dispersion scale for transport through dense cubic packed spheres with vortices," Geochem., Geophys., Geosyst. 10(12), Q12014, doi:10.1029/2009GC002593 (2009).

${ }^{37}$ J. E. Garcia and K. Pruess, "Flow instabilities during injection of $\mathrm{CO} 2$ into saline aquifers," in Proceedings of Tough Symposium 2003 (Lawrence Berkeley National Laboratory, 2003).

${ }^{38}$ J. G. I. Hellström, P. J. P. Jonsson, and T. S. Lundström, "Laminar and turbulent flow through an array of cylinders," J. Porous Media 13(12), 1073-1085 (2010).

${ }^{39}$ T. Masuoka, Y. Takatsu, and T. Inoue, "Chaotic behavior and transition to turbulence in porous media," Nanoscale Microscale Thermophys. Eng. 6(4), 347-357 (2002).

${ }^{40}$ C. Pozrikidis, "Creeping flow in two-dimensional channels," J. Fluid Mech. 180, 495-514 (1987).

${ }^{41}$ A. E. Malevich, V. V. Mityushev, and P. M. Adler, "Stokes flow through a channel with wavy walls," Acta Mech. 182(3-4), 151-182 (2006).

${ }^{42}$ H. K. Moffatt, "Viscous and resistive eddies near a sharp corner," J. Fluid Mech. 18, 1-18 (1963).

${ }^{43}$ R. Haggerty and S. M. Gorelick, "Multiple-rate mass transfer for modeling diffusion and surface reactions in media with pore-scale heterogeneity," Water Resour. Res. 31(10), 2383, doi:10.1029/95WR10583 (1995).

${ }^{44}$ J. Carrera, X. Sánchez-Vila, I. Benet, A. Medina, G. Galarza, and J. Guimerà, "On matrix diffusion: Formulations, solution methods and qualitative effects," Hydrogeol. J. 6(1), 178-190 (1998).

${ }^{45}$ L. D. Donado, X. Sanchez-Vila, M. Dentz, J. Carrera, and D. Bolster, "Multicomponent reactive transport in multicontinuum media," Water Resour. Res. 45(11), W11402, doi:10.1029/2008WR006823 (2009).

${ }^{46}$ R. Zimmerman, S. Kumar, and G. Bodvarsson, "Lubrication theory analysis of the permeability of rough-walled fractures," Int. J. Rock Mech. Min. Sci. Geomech. Abstr. 28(4), 325-331 (1991).

${ }^{47}$ S. R. Brown, "Simple mathematical model of a rough fracture," J. Geophys. Res. 100(B4), 5941-5952, doi:10.1029/94JB03262 (1995).

${ }^{48}$ T. Le Borgne, D. Bolster, M. Dentz, P. de Anna, and A. Tartakovsky, "Effective pore-scale dispersion upscaling with a correlated continuous time random walk approach," Water Resour. Res. 47(12), 1-10, doi:10.1029/2011WR010457 (2011).

${ }^{49}$ J. Bear, Dynamics of Fluids in Porous Media, Elsevier ed. (Dover, New York, 1972). 